\section{Vecabrutinib inhibits B-cell receptor signal transduction in chronic lymphocytic leukemia cell types with wild-type or mutant Bruton tyrosine kinase}

Ibrutinib as monotherapy or in combination has transformed the treatment landscape of chronic lymphocytic leukemia (CLL). ${ }^{1,2}$ This drug covalently tethers to the cysteine-481 residue in Bruton tyrosine kinase (BTK), which is a pivotal enzyme in the $\mathrm{B}$-cell receptor (BCR) pathway. ${ }^{3}$ Ibrutinib treatment results in long-term overall survival in patients with CLL; however, disease can relapse, particularly in previously treated patients. At a median of 3.4 years of follow-up, the cumulative incidence of progression was $19 \%$, and $85 \%$ of these patients had acquired mutations of BTK or PLCG2., ${ }^{4,5}$ The predominant BTK mutation is cysteine to serine $\left(\mathrm{BTK}^{\mathrm{C} 4815}\right)$, with the second most frequent alteration being cysteine to arginine $\left(\mathrm{BTK}^{\mathrm{C} 481 \mathrm{R}}\right)$, both of which preclude covalent bond formation by ibrutinib, resulting in resistance to this drug. ${ }^{4}$ Reversible BTK inhibitors, such as vecabrutinib, have been developed that bind to BTK and maintain inhibitory activity against WT and mutant BTK. In this study, we characterized the activity of vecabrutinib on the BCR pathway using a CLL cell line model system engineered to overexpress BTK C481 WT or two mutant variants, $\mathrm{BTK}^{\mathrm{C} 481 \mathrm{~S}}$ and $\mathrm{BTK} \mathrm{K}^{\mathrm{C} 481 \mathrm{R}}$. ${ }^{6} \mathrm{We}$ also used primary CLL cells resistant to ibrutinib to further extend our investigations of vecabrutinib. A phase $1 \mathrm{~b}$ clinical trial was recently completed in B-cell malignancies in which vecabrutinib was well-tolerated with some evidence of activity including in CLL patients with the C481S mutation.?

Vecabrutinib is a highly selective reversible BTK inhibitor (half maximal inhibitory concentration $\left[\mathrm{IC}_{50}\right]=$ $3 \mathrm{nM}$ ). In a panel of 234 kinases and kinase variants, vecabrutinib demonstrated a biochemical $\mathrm{IC}_{50}$ of $<100$ $\mathrm{nM}$ for seven kinases (Figure 1A; Online Supplementary Figure $S 1 A, B)$. The $\mathrm{IC}_{50}$ of vecabrutinib against WT BTK was similar to that of ibrutinib ${ }^{3}$ while it was more potent than ibrutinib on ITK and TEC kinases. In a direct kinase assay, vecabrutinib inhibited WT and mutant $\mathrm{C} 481 \mathrm{~S}$ variants with similar potency. Data from healthy donors' whole blood $(n=145)$ further established the potency of vecabrutinib in inhibiting BTK, although there was a high degree of variability (mean \pm standard deviation values, $50 \mathrm{nM} \pm 39 \mathrm{nM}$, range $2.8 \mathrm{nM}-216 \mathrm{nM}$ ) (Figure 1B). Vecabrutinib inhibited phosphorylation of the BTK downstream target PLC $\gamma 2$ in Ramos Burkitt lymphoma cells with $\mathrm{IC}_{50}$ values of $13 \pm 6 \mathrm{nM}$ (Figure 1C). Collectively, these data suggest that vecabrutinib inhibits phosphorylation of BTK and of PLC $\gamma 2$ at nanomolar concentrations.

In MEC-1, a CLL cell line, treatment with both vecabrutinib and ibrutinib did not alter cell cycle profiles and resulted in $15-20 \%$ cell death at $1 \mu \mathrm{M}$ (Online Supplementary Figure S1C-E). Vecabrutinib decreased BTK phosphorylation at a dose of $0.1 \mu \mathrm{M}$. Consistent with the observed decline in BTK phosphorylation, decreases in phosphorylation of PLC $\gamma 2$ and ERK were also observed. Phospho-S6 levels did not change in the MEC-1 cell line after treatment with vecabrutinib (Online Supplementary Figure S1F).

To mimic ibrutinib resistance, we transduced MEC-1 to generate cell lines that stably expressed green fluorescent protein and overexpressed either WT BTK $\left(\mathrm{BTK}^{\mathrm{WT}}\right)$ or the mutated variant $\mathrm{C} 481 \mathrm{~S}\left(\mathrm{BTK}^{\mathrm{C} 481 \mathrm{~S}}\right)$, or $\mathrm{C} 481 \mathrm{R}\left(\mathrm{BTK}^{\mathrm{C} 481 \mathrm{R}}\right)$ BTK. ${ }^{6}$ Cell death induced by ibrutinib or acalabrutinib has been limited when the drugs have been tested in vitro in B-cell lines or primary CLL cells, ${ }^{8,9}$ and similarly, vecabrutinib did not affect cell viability or cell cycle profile (Online Supplementary Figure S2A-D). Several known proteins within the BCR signal transduction pathway, including BTK, can be used as biomarkers to monitor ibrutinib response or biological activity, including ERK and S6. ${ }^{8,10}$ We evaluated these proteins by immunoblot for phosphorylated proteins to show response to vecabrutinib and ibrutinib in cell lines that harbor WT overexpression or mutant BTK overexpression. Vecabrutinib at a dose of $1 \mu \mathrm{M}$ decreased phospho-ERK more effectively than ibrutinib did in ibrutinib-resistant MEC-1 cells that overexpress mutant BTK (Figure 1D-F). This was observed in both $\mathrm{BTK}^{\mathrm{C} 481 \mathrm{~S}}$ and $\mathrm{BTK} \mathrm{K}^{\mathrm{C} 481 \mathrm{R}}$ variants. Changes in phospho-ERK were consistent with those in our previous study, in which we showed that phospho-ERK is a superior biomarker to determine ibrutinib response upon overexpression of mutant BTK in MEC- $1^{6}$ (Figure 1E, F).

It is important to note that ibrutinib also decreased phospho-proteins in cells with mutated BTK. There are two explanations for this finding. First, the cell lines express endogenous WT BTK and second, ibrutinib can bind reversibly to BTK, albeit with reduced potency. In the clinic, ibrutinib's poor pharmacokinetic properties (peak plasma level along with initial and terminal elimination half-lives) preclude activity as a noncovalent inhibitor. Vecabrutinib treatment at a dose of $1 \mu \mathrm{M}$ decreased $\mathrm{Bcl}-2$ levels in cells harboring $\mathrm{BTK}^{\mathrm{C} 481 \mathrm{~S}}$ and $\mathrm{Mcl}-1$ in cells overexpressing $\mathrm{BTK}^{\mathrm{C} 481 \mathrm{R}}$ (Online Supplementary Figure S2E-G).

To evaluate protein changes more extensively as well as to compare the effects of vecabrutinib with those of ibrutinib, we compared protein profiles using the reversephase protein array (RPPA). Since we observed the largest effect of vecabrutinib after treatment at $1 \mu \mathrm{M}$, in all cell lines we compared this concentration with samples treated with dimethylsulfoxide (DMSO) vehicle. The top ten canonical pathways were identified by Ingenuity Pathway Analysis (Online Supplementary Figure S3A-C). Several pathways were commonly affected in all three cell types. The extent of change and significance were different (Figure 2A). The top three canonical pathways with maximal change after vecabrutinib in cells with $\mathrm{BTK}^{\mathrm{WT}}$ overexpression were FLT3 signaling in hematopoietic progenitor cells, EGF and HGF signaling (Online Supplementary Figure S3A), whereas in cells with $\mathrm{BTK}^{\mathrm{C} 481 \mathrm{~S}}$ overexpression they were HGF signaling, regulation of epithelial-mesenchymal transition by growth factors pathway and B-cell receptor signaling (Online Supplementary Figure $S 3 B$ ) and in cells with $\mathrm{BTK}^{\mathrm{C} 481 \mathrm{R}}$ overexpression they were epithelial-mesenchymal transition, ERK/MAPK signaling, and a senescence pathway (Online Supplementary Figure S3C). We also classified the types of target proteins using pie charts (Online Supplementary Figure $S 3 D-F)$. Kinase and transcription regulator protein groups constituted $>60 \%$ of proteins affected by vecabrutinib in all BTK subtypes.

BCR pathway inhibition generally affects signal transduction, measured as phospho-proteins, proteins involved in transcription factors, cell proliferation, B-cell proteins, and apoptosis. Among the 258 proteins evaluated by RPPA, eight phospho-proteins and five other proteins were affected by vecabrutinib and ibrutinib (Figure 2B). SHP-2 (PTPN11), a phosphatase that plays a critical role at several junctures in the BCR pathway, has been shown to interact with many proteins. ${ }^{11}$ The tyrosyl phosphorylation of this protein has been shown to be 
A

\begin{tabular}{|l|c|c|}
\hline $\begin{array}{l}\text { TEC Family } \\
\text { Kinases }\end{array}$ & $\begin{array}{c}\text { Vecabrutinib } \\
\text { IC }_{50} \mathbf{n M}\end{array}$ & $\begin{array}{c}\text { Ibrutinib } \\
\text { IC }_{50} \mathbf{n M}\end{array}$ \\
\hline BTK & 3 & 0.5 \\
\hline ITK & 14 & 10.7 \\
\hline TEC & 14 & 78 \\
\hline TXK & 474 & 2 \\
\hline BMK & 224 & 0.8 \\
\hline Other Kinases & & \\
\hline LCK & 8 & 33.2 \\
\hline C-Src & 84 & 171 \\
\hline BLK & 23 & 0.5 \\
\hline NEK11 & 90 & NR \\
\hline EGFR & $>1000(6644)$ & 5.6 \\
\hline ERBB2 & 450 & 9.4 \\
\hline ERBB4 & 317 & 3.4 \\
\hline CSK & $>1000$ & 2.3 \\
\hline FGR & $>1000$ & 2.3 \\
\hline BRK & $>1000$ & 3.3 \\
\hline HCK & 276 & 3.7 \\
\hline YES & 402 & 6.5 \\
\hline JAK3 & $>1000$ & 16.1 \\
\hline FRK & $>1000$ & 29.2 \\
\hline RET & $>1000$ & 36.5 \\
\hline FLT3 & $>1000$ & 73 \\
\hline ABL & $>1000$ & 86 \\
\hline FYN & 651 & 96 \\
\hline
\end{tabular}

B

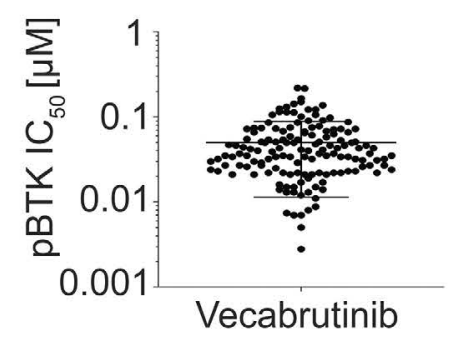

C

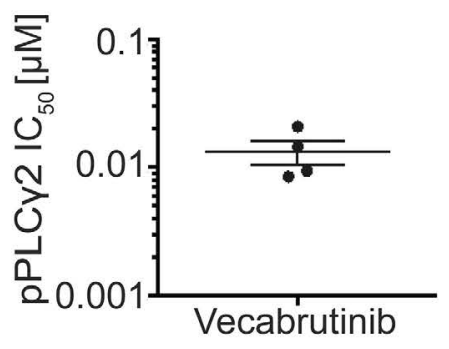

D BTKWT $^{W T}$

Vecabrutinib Ibrutinib

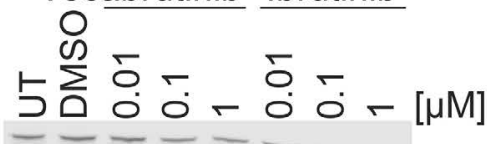

pBTK

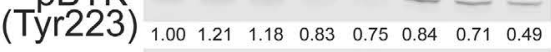

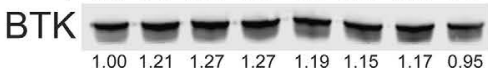
pERK

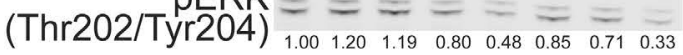

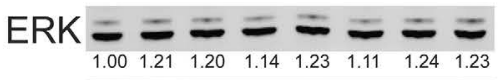

Vinculin - - - - - -

E

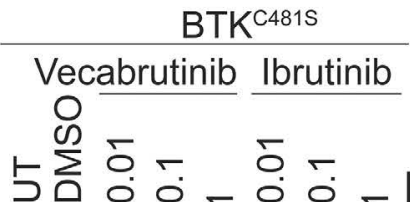
$[\mu \mathrm{M}]$

pBTK - - - - - - -

$\begin{array}{lllllllll}\text { (Tyr223) } & 1.00 & 1.21 & 1.23 & 0.90 & 0.65 & 1.00 & 0.88 & 0.30\end{array}$

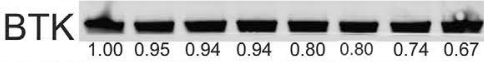
$\mathrm{pERK}$

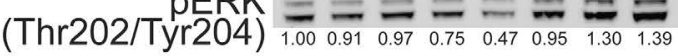

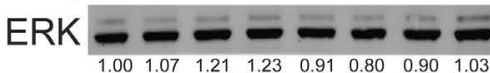

Vinculin - - - - - - -

$\mathbf{F}$

\section{$\mathrm{BTK}^{\mathrm{C} 481 \mathrm{R}}$}

Vecabrutinib Ibrutinib

$$
\text { 与 } \sum_{0}^{0} \dot{0} \overline{0}-\overline{0} \cdot
$$

$\begin{array}{lllllllllllll}\text { (Tyr223) } & 1.00 & 1.40 & 1.38 & 1.18 & 1.82 & 2.67 & 3.72 & 1.09\end{array}$

BTK - - - - - - $\begin{array}{llllllll}1.00 & 1.22 & 1.48 & 1.49 & 1.56 & 1.85 & 3.05 & 1.55\end{array}$ $\mathrm{pERK}=====$

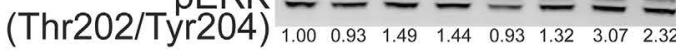

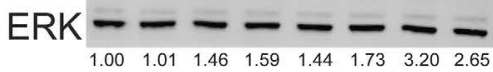

Vinculin - - - - - - -

Figure 1. Vecabrutinib kinome profile and effect on B-cell receptor pathway protein phosphorylation. (A) Vecabrutinib and ibrutinib kinase inhibition profiles. Vecabrutinib kinase selectivity and kinase inhibitory activities were evaluated in the KinaseProfilerTM panel of 234 individual kinases (Eurofins Pharma Discovery Services, Dundee, UK). For TEC and LCK, vecabrutinib activity was assessed against the activated kinase. (B) Phospho-BTK inhibition in human whole blood ex vivo. Peripheral blood mononuclear cells were isolated from peripheral blood of healthy donors and were incubated with 0.3 to $10,000 \mathrm{nM}$ vecabrutinib for $0.5 \mathrm{~h}$. Experiments were conducted on samples from 145 individuals to calculate $\mathrm{IC}_{50}$ values. Mean and standard deviation (SD) values are shown with horizontal lines and error bars. (C) Phospho-PLC 2 inhibition in the Ramos cell line. Cells were incubated with 0.3 to 10,000 nM vecabrutinib. Experiments were conducted on four biological replicates in the Ramos cell line to calculate $\mathrm{IC}_{50}$ values. Mean and SD values are shown with horizontal lines and error bars. (D-F). Effects of vecabrutinib on BCR pathway proteins in MEC-1 cells that overexpress wild-type or mutant BTK. Green fluorescence protein (GFP)-labeled MEC-1 cell lines that stably overexpress wild-type BTK $\left(\mathrm{BTK}^{\mathrm{WT}}\right)$ and mutant BTK (BTK ${ }^{\mathrm{C} 81 \mathrm{~S}}$ or $\mathrm{BTK}^{\mathrm{C} 481 \mathrm{R}}$ ) were generated by using standard lentiviral transfection methods. Cells were sorted by a BD FACSAria (BD Biosciences) for the enrichment of transduced GFP ${ }^{+}$cell populations in each cell line. For all experiments, $>75 \%$ GFPpositive cell populations were used. Cells were treated with indicated concentrations of vecabrutinib or ibrutinib and incubated for $24 \mathrm{~h}$. Protein extracts were subjected to immunoblot assays to determine levels of phospho-BTK (Y223), BTK, phospho-ERK (T202/Y204), ERK in MEC-1 cells overexpressing (D) BTK ${ }^{\text {WT }}$, (E) $\mathrm{BTK}^{\mathrm{C} 481 \mathrm{~s}}$, and (F) BTK ${ }^{\mathrm{C} 481 \mathrm{R}}$ cells. Vinculin was used as the loading control. 
A

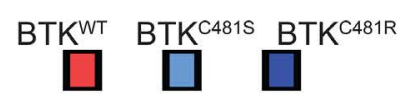

PTEN signaling

ErbB2-ErbB3 signaling

MSP-RON signaling in cancer cells pathway

Antiproliferative role of TOB in T cell signaling

ERK/MAPK signaling

Thrombopoietin signaling

14-3-3 mediated signaling

IL-6 signaling

Apoptosis signaling

RAR activation

Regulation of epithelial-mesenchymal

B cell receptor signaling

GM-CSF signaling

Neuregulin signaling

IGF-1 signaling

Senescence pathway

HGF signaling

EGF signaling

FLT3 signaling

in hematopoietic

progenitor cells 0

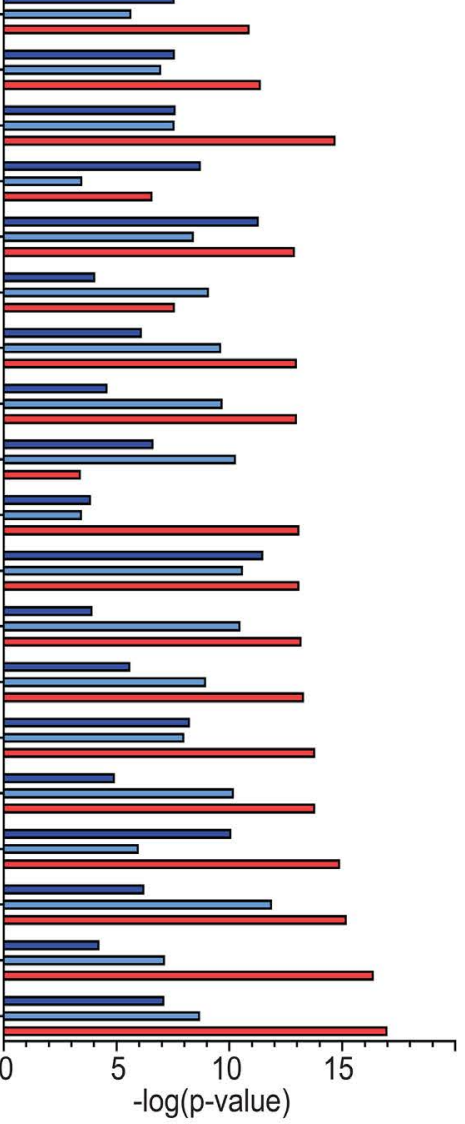

음

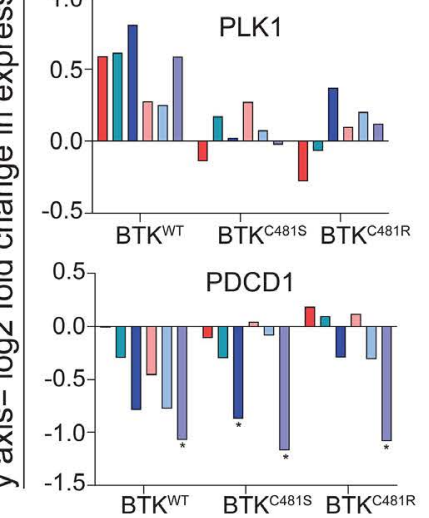

B

$$
\bigwedge \begin{aligned}
& \square \text { Vecabrutinib } 0.01 \mu \mathrm{M} \\
& \square \text { Vecabrutinib } 0.1 \mu \mathrm{M} \\
& \square \text { Vecabrutinib } 1 \mu \mathrm{M} \\
& \square \text { Ibrutinib } 0.01 \mu \mathrm{M} \\
& \square \text { Ibrutinib } 0.1 \mu \mathrm{M} \\
& \square \text { Ibrutinib } 1 \mu \mathrm{M}
\end{aligned}
$$
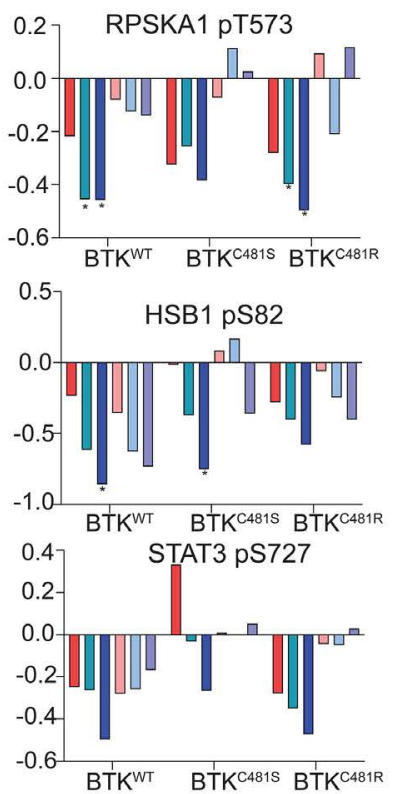

$1.5 \quad * \quad$ ETS1

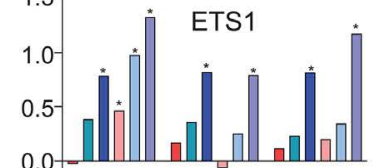

0.0

$-0.5$

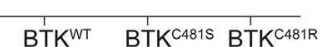

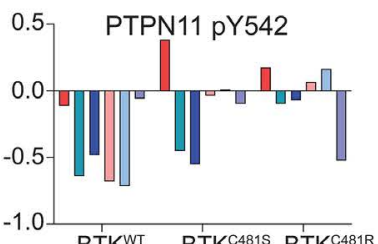

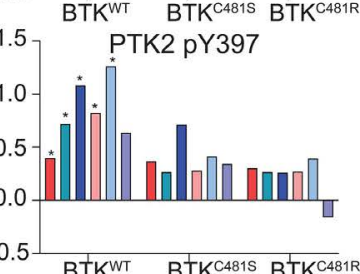

0.51 MAPK 1 pT202/Y204

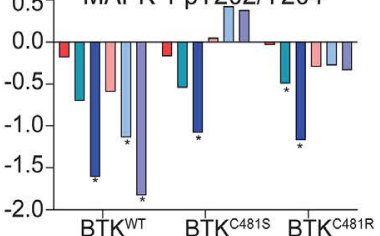

0.4
0.2 $\quad$ STAT3 pY705

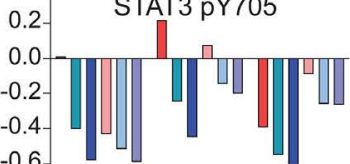

$-0.6$

BTK $^{\mathrm{WT}} \mathrm{BTK}^{\mathrm{I}}{ }^{\mathrm{C} 481 \mathrm{~S}}$ BTKK $^{\mathrm{C} 481 \mathrm{R}}$

1.07

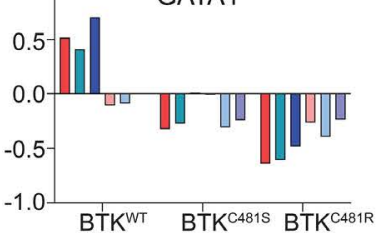

${ }^{1.0} 0_{7} \quad$ CDKN1B pT198

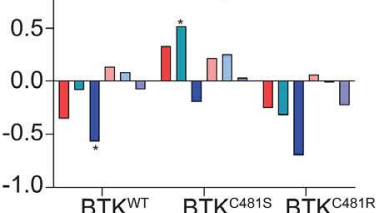

BTKWT BTKC481S BTKC481R

0.6 Cleaved caspase 7

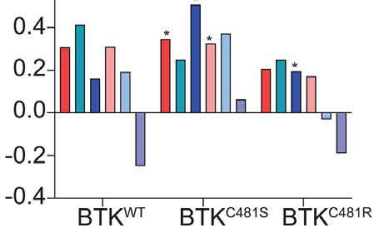

Figure 2. Effect of vecabrutinib treatment on functional protein profiles of wild-type and mutant BTK-overexpressing MEC-1 cells. (A) Effect of vecabrutinib on common pathways in all three cell lines. Exponentially growing MEC-1 cells with either wild-type or mutant BTK were treated for $24 \mathrm{~h}$ with vecabrutinib at $1 \mathrm{\mu M}$. Experiments were performed in biological triplicates ( $n=3 /$ cell line). At the end point, cells were collected, and protein was extracted and was subjected to the reverse-phase protein array (RPPA) that included 258 antibodies. Changes in expression $\left(\mathrm{RPPA}_{\text {treatment }}[1 \mu \mathrm{M}]-\mathrm{RPPA}_{\mathrm{DMSO}_{\mathrm{N}}}\right)$ values were used to determine the top canonical pathways identified with Ingenuity Pathway Analysis and associated with BTK ${ }^{\mathrm{WT}}$ (red bars), BTK ${ }^{\mathrm{C} 81 \mathrm{~S}}$ (light blue bars), and BTK ${ }^{\mathrm{C} 481 \mathrm{R}}$ (navy blue bars) cells. (B) Effect of vecabrutinib treatment on proteins in wild-type and mutant BTK-overexpressing MEC-1 cells. Exponentially growing MEC-1 cells with either wild-type or mutant BTK were treated with three concentrations of vecabrutinib or ibrutinib and then their proteins were extracted and subjected to RPPA assays as described in the Methods section. Graphs were generated using the $\log _{2}$ fold change in expression (RPPA treatment $^{-R P P A_{D M s o}}$ ) values obtained by analysis of the RPPA data. Legends used in all graphs in Figure 2B are included in the upper left corner. Gene names and phosphorylation sites are indicated on top of each figure. Statistical comparisons were made between dimethylsulfoxide (DMSO)- versus ibrutinib- or vecabrutinib-treated cells, and asterisks depict $P$ values $<0.05$. 
A

\begin{tabular}{|l|c|l|}
\hline Pt no & $\begin{array}{c}\text { Prior BTKi } \\
\text { Treatment }\end{array}$ & $\begin{array}{c}\text { BTK mutation } \\
\text { status }\end{array}$ \\
\hline Pt 1 & Ibrutinib & WT \\
\hline Pt 2 & Ibrutinib & T474F \\
\hline Pt 3 & Ibrutinib & C481S, C481R \\
\hline Pt 4 & Acalabrutinib & C481S, T474I \\
\hline Pt 5 & Acalabrutinib & C481S, T474I \\
\hline
\end{tabular}

C

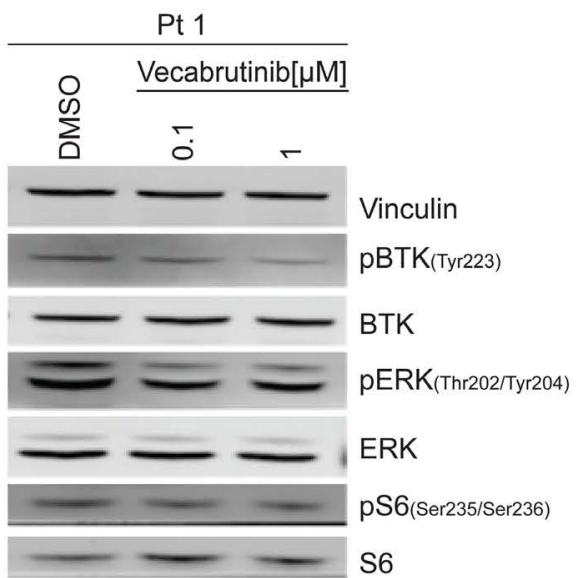

B

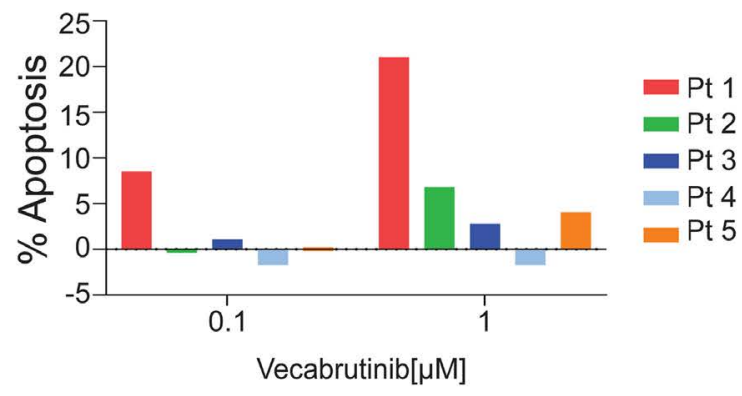

D

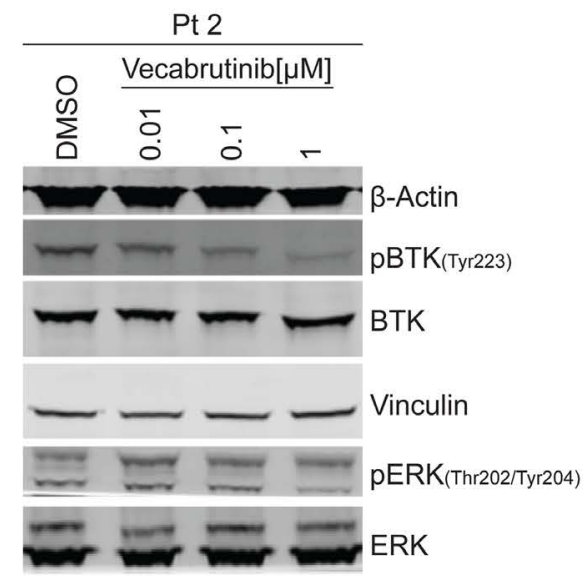

E

Pt 3

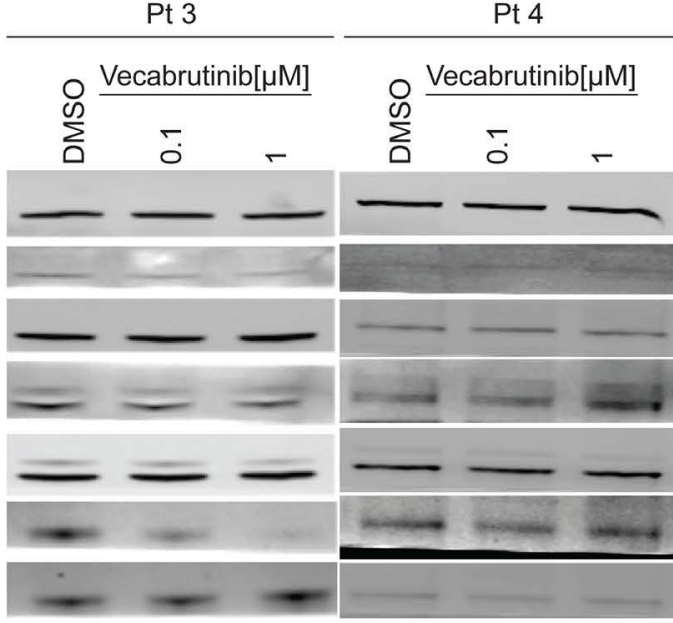

Pt 5

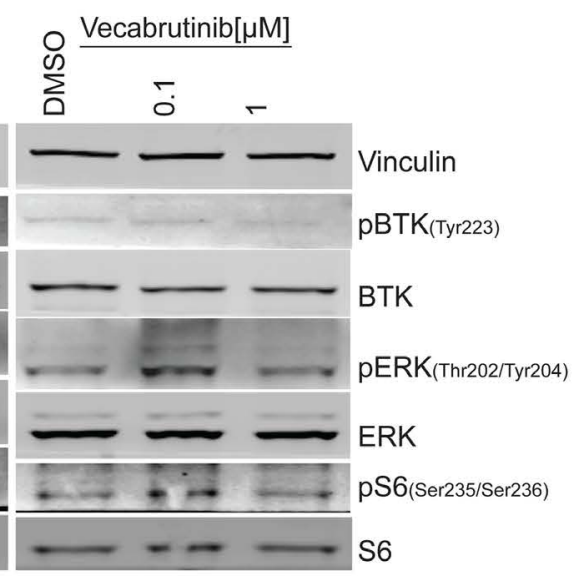

Figure 3. Inhibition of the B-cell receptor pathway in chronic lymphocytic leukemia cells from patients with wild-type or mutant BTK. For blood sample collections, patients $(n=5)$ provided written informed consent for the protocol, which was approved by the Institutional Review Board of The University of Texas MD Anderson Cancer Center, in accordance with the Declaration of Helsinki. Blood samples were collected into Vacutainer glass green-top blood collection tubes and cells were isolated by Ficoll-Hypaque density centrifugation and incubated with vecabrutinib at two or three concentrations $(0.01,0.1$, and $1 \mu \mathrm{M})$ for $24 \mathrm{~h}$. (A) Table presenting the patients' numbers and BTK mutation status for five patients with chronic lymphocytic leukemia (CLL). BTK mutations were identified in patients' samples using End CLL or End Lymphoma panels that included C481F, C481R, C481S, L528W, T474I, and T474F mutations. The same patients' numbers are used in Figure 3B-E. (B) Apoptotic cell death in primary CLL lymphocytes of five patients. Freshly isolated cells (patients 1-3) or cryopreserved cells (patients 4 and 5 ) were incubated for $24 \mathrm{~h}$ with indicated concentrations of vecabrutinib. Cells were stained with annexin V-FITC and propidium iodide (PI) and apoptotic cells were determined with flow cytometry. Cell death in dimethylsulfoxide (DMSO)-treated samples was subtracted from that of treated samples. Percent apoptosis in DMSO was $14.6,0.3,3.3,73$, and 41.9 in patients 1 to 5 , respectively. (C-E) Effect of vecabrutinib on B-cell receptor pathway proteins. Protein extracts were subjected to immunoblot assays to determine levels of phospho-BTK (Y223), BTK, phospho-ERK (T202/Y204), ERK, phospho-S6 (Ser235/236), and S6. (C, D) Patients with BTK ${ }^{\mathrm{WT}}$ or BTK ${ }^{\mathrm{T} 74 \mathrm{~F}}$ mutant CLL cells, (E) Patients with BTK ${ }^{\mathrm{C} 481 \mathrm{1S}}$ and BTK ${ }^{\mathrm{C} 481 \mathrm{R}}$ variants. $^{\text {B }}$ 
essential for the activation of ERK, a downstream molecule in BCR signaling. ${ }^{12}$ Compared to DMSO-treated cells, BTK inhibition substantially decreased phosphoSHP2(Y542) in WT cells, whereas only vecabrutinib specifically inhibited SHP-2 phosphorylation in both mutant cell lines (Figure 2B).

Other proteins that showed a change after drug treat-

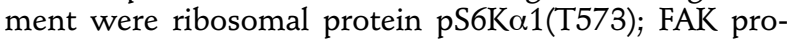
tein pPTK2(Y397); pHSB1(S82); transcription factor pERK1/2(T202/Y204); transcription factors pSTAT3(S727); pSTAT3(Y705); transcription factors ETS1 and GATA1; cell cycle proteins PLK1, and phosphop27(T198); B-cell protein PD1; and cleaved caspase 7 (Figure 2B). In general, among WT and mutant variants, changes in these proteins were more pronounced in WT cells than in mutants.

Of the two drugs, vecabrutinib produced deeper or similar responses compared to those to ibrutinib in WT cells for all proteins (Figure 2B). Consistent with a prior report, ${ }^{12}$ treatment with ibrutinib and vecabrutinib caused a 2-log decrease in phospho-ERK in $\mathrm{BTK}^{\mathrm{WT}}$ cells after treatment. Furthermore, vecabrutinib treatment resulted in a 1-log decline in cells harboring mutant BTK, whereas ibrutinib had no effect (Figure 2B). A hallmark protein that is affected by ibrutinib is p70S6K or S6 kinase. The target protein of this kinase is S6 ribosomal protein, which initiates protein synthesis upon phosphorylation. Impressively, compared to ibrutinib, vecabrutinib profoundly decreased phospho-S6K in all three cell types. Among all proteins, phosphorylated ERK and S6K were consistently and substantially decreased in all three cell types. These two proteins may serve as biomarkers for the effect of vecabrutinib (Figure 2B).

Ibrutinib produced a larger decrease in PD1 levels than vecabrutinib did. Parallel to PD1 protein levels, serine(727) and tyrosine(705) phosphorylation of STAT3 has been shown to be reduced in CLL cells after ibrutinib treatment. ${ }^{13}$ STAT3 and STAT5 activation through phosphorylation has been associated with inflammation and carcinogenesis. ${ }^{14}$ STAT3 is constitutively active in CLL cells, but this activation is mitigated by ibrutinib treatment. ${ }^{13,15}$ Vecabrutinib treatment decreased Y705 and S727 phosphorylation on STAT3 in WT and mutant cell lines. Interestingly, in mutant cells, a decline in S727 phosphorylation occurred only with vecabrutinib (Figure 2B). Although extensive cell death was not seen with either of these drugs, RPPA data revealed that vecabrutinib treatment increased cleaved caspase 7 in all transduced cell lines.

Finally, we tested vecabrutinib in primary CLL cells from five patients with either WT or mutant BTK (Figure $3 \mathrm{~A})$. Cell death ranged from 0 up to $21 \%$ after 24 hours of vecabrutinib, being higher in $\mathrm{BTK}^{\mathrm{WT}}$ than in $\mathrm{BTK}_{\text {mutant }}$ samples (Figure 3B). BTK with C481S and C481R alterations had the lowest apoptosis. In concert, immunoblot results showed that vecabrutinib inhibited BCR pathway (phosphorylation of BTK, ERK, and S6) signaling in $\mathrm{BTK}^{\mathrm{WT}}$ and in $\mathrm{BTK}^{\mathrm{T} 474 \mathrm{~F}}$ (gate-keeper mutation) (Figure 3C, D). Consistent with the RPPA data, cells expressing the $\mathrm{BTK}^{\mathrm{C} 481 \mathrm{~S}}$ and $\mathrm{BTK}^{\mathrm{C} 481 \mathrm{R}}$ variants had minor changes (patient 3). In patients 4 and 5, phospho-ERK either remained the same or increased; these two patients had C481S (catalytic domain) and T474I (gate-keeper) double mutations (Figure 3E). Super-resistance to irreversible BTK inhibitors or variable sensitivity to reversible noncovalent BTK inhibitors has been reported for cells harboring T474 variants along with the cysteine 481 substitution. 16

In summary, our study provides a nonclinical character- ization of vecabrutinib. This reversible BTK inhibitor is selective in kinome profiling and inhibits phosphorylation of BTK and PLC $\gamma 2$ in preclinical whole-cell assays. Ibrutinib-sensitive and -resistant model systems demonstrated inhibition of the BCR signal transduction cascade in both BTK ${ }^{\mathrm{WT}}$ and BTK ${ }^{\text {mutant }}$ B-cell lines. ${ }^{6}$ Data were consistent in CLL cells, but the impact on $\mathrm{BTK}^{\mathrm{C} 481 \mathrm{~S}}$ and $\mathrm{BTK}^{\mathrm{C} 481 \mathrm{R}}$ was modest in primary cells. The results of a clinical trial of vecabrutinib in B-cell malignancies will be reported soon. ${ }^{7}$

Burcu Aslan, ${ }^{1}$ Stefan Edward Hubner, Judith A. Fox, ${ }^{3}$ Pietro Taverna, ${ }^{3}$ William G. Wierda, ${ }^{2}$ Steven M. Kornblau ${ }^{2}$ and Varsha Gandhi, ${ }^{1,2}$

${ }^{1}$ Department of Experimental Therapeutics, The University of Texas MD Anderson Cancer Center, Houston, TX; ${ }^{2}$ Department of Leukemia, The University of Texas MD Anderson Cancer Center, Houston, TX and ${ }^{3}$ Sunesis Pharmaceuticals, Inc., South San Francisco, CA, USA

Correspondence:

VARSHA GANDHI -vgandhi@mdanderson.org.

doi:10.3324/haematol.2021.279158

Received: May 6, 2021.

Accepted: September 1, 2021.

Pre-published: September 9, 2021

Disclosures: related to this work, VG has sponsored research agreements with Sunesis Pharmaceuticals and Loxo Oncology. Outside of this work, VG has received research support from AbbVie, Acerta, AstraZeneca, ClearCreek Bio, Dava Oncology, Gilead, and Pharmacyclics. JAF and PT were employees of Sunesis

Pharmaceuticals. The other authors have no conflicts of interest.

Contributions: BA designed and performed the experiments, analyzed the results, and wrote the first draft of the manuscript; SEH performed the bioinformatics analyses of the RPPA data; $J F$ and PT contributed other nonclinical and clinical investigations of vecabrutinib, provided data that are included in Figure 1, and reviewed the manuscript; WGW identified ibrutinib-resistant patients and provided samples; SMK supervised SEH and analyzed RPPA data; $V G$ conceptualized and supervised the research, obtained funding, analyzed the data, and wrote and revised the manuscript.

Acknowledgments: the authors thank Drs. Joseph R. Marszalek, Christopher P. Vellano, Michael Peoples, and Mikhila Mahendra for the creation of MEC-1 cell lines that overexpress WT and mutant BTK. The authors thank LaKesla Iles for technical assistance and Tamara Locke, Scientific Editor, Research Medical Library, for editing this article.

Funding: this work was supported in part by The University of Texas MD Anderson Cancer Center Moon Shot Program, a Sponsored Research Agreement from Sunesis Pharmaceuticals, and by the NIH/NCI through award number P30CA016672.

\section{References}

1. Burger JA. Treatment of chronic lymphocytic leukemia. N Engl J Med. 2020:383(5):460-473

2. Timofeeva N, Gandhi V. Ibrutinib combinations in CLL therapy: scientific rationale and clinical results. Blood Cancer J. 2021;11(4):79.

3. Honigberg LA, Smith AM, Sirisawad M, et al. The Bruton tyrosine kinase inhibitor PCI-32765 blocks B-cell activation and is efficacious in models of autoimmune disease and B-cell malignancy. Proc Natl Acad Sci U S A. 2010;107(29):13075-13080.

4. Woyach JA, Ruppert AS, Guinn D, et al. BTKC481S-mediated resistance to ibrutinib in chronic lymphocytic leukemia. J Clin Oncol. 2017; 35(13):1437

5. Burger JA, Landau DA, Taylor-Weiner A, et al. Clonal evolution in patients with chronic lymphocytic leukaemia developing resistance to BTK inhibition. Nat Commun. 2016;7(1):1-13

6. Aslan B, Kismali G, Chen LS, et al. Development and characterization of prototypes for in vitro and in vivo mouse models of ibrutinib-resistant 
CLL. Blood Adv. 2021:5(16):3134-3146.

7. Allan JN, Patel K, Mato AR, et al. Ongoing results of a phase $1 \mathrm{~B} / 2$ doseescalation and cohort-expansion study of the selective, noncovalent, reversible bruton's tyrosine kinase inhibitor, vecabrutinib, in B-cell malignancies. Blood. 2019;134(Supplement 1):3041.

8. Herman SE, Mustafa RZ, Gyamfi JA, et al. Ibrutinib inhibits BCR and $\mathrm{NF}-\kappa \mathrm{B}$ signaling and reduces tumor proliferation in tissue-resident cells of patients with CLL. Blood. 2014;123(21):3286-3295.

9. Patel V, Balakrishnan K, Bibikova E, et al. Comparison of acalabrutinib, a selective Bruton tyrosine kinase inhibitor, with ibrutinib in chronic lymphocytic leukemia cells. Clinl Cancer Res. 2017;23(14):3734-3743.

10. Ponader S, Burger JA. Bruton's tyrosine kinase: from X-linked agammaglobulinemia toward targeted therapy for B-cell malignancies. J Clin Oncol. 2014;32(17):1830.

11. Neel BG, Gu H, Pao L. The 'Shp'ing news: SH2 domain-containing tyrosine phosphatases in cell signaling. Trends Biochem Sci. 2003;28(6):284 293
12. Araki T, Nawa H, Neel BG. Tyrosyl phosphorylation of Shp2 is required for normal ERK activation in response to some, but not all, growth factors. J Biol Chem. 2003;278(43):41677-41684

13. Kondo K, Shaim H, Thompson PA, et al. Ibrutinib modulates the immunosuppressive CLL microenvironment through STAT3-mediated suppression of regulatory B-cell function and inhibition of the PD-1/PDL1 pathway. Leukemia. 2018;32(4):960-970.

14. Loh C-Y, Arya A, Naema AF, et al. Signal transducer and activator of transcription (STATs) proteins in cancer and inflammation: functions and therapeutic implication. Front Oncol. 2019;9:48.

15. Rui L, Drennan AC, Ceribelli M, et al. Epigenetic gene regulation by Janus kinase 1 in diffuse large B-cell lymphoma. Proc Natl Acad Sci U S A. 2016;113(46):E7260-E7267.

16. Estupiñán HY, Wang $\mathrm{Q}$, Berglöf $\mathrm{A}$, et al. BTK gatekeeper residue variation combined with cysteine 481 substitution causes super-resistance to irreversible inhibitors acalabrutinib, ibrutinib and zanubrutinib. Leukemia. 2021;35(5):1317-1329 